\title{
HAEMATOLOGICAL PARAMETERS AND C-REACTIVE PROTEIN IN PREDICTION OF DISEASE SEVERITY AND MORTALITY IN PATIENTS WITH SEPSIS
}

\author{
Sehveta Mustafić ${ }^{1,2 *}$, Selmira Brkić ${ }^{2}$, Alma Mujić-Ibralic ${ }^{2,3}$ \\ ${ }^{1}$ Department of Laboratory Diagnostics, University Clinical Centre Tuzla, Tuzla, Bosnia and \\ Herzegovina \\ ${ }^{2}$ Faculty of Medicine, University in Tuzla, Tuzla, Bosnia and Herzegovina \\ ${ }^{3}$ Department of Internal Medicine, Community Health Care Centre Zivinice, Zivinice, Bosnia \\ and Herzegovina
}

Original Scientific Article

Received: 23/03/2021

Accepted: 13/05/2021

\begin{abstract}
Sepsis is a life-threatening condition characterized by a systemic inflammatory response of the body caused by a severe infection. The aim of this study was to examine the importance of hematological parameters and c-reactive protein (CRP) in the diagnosis of sepsis, assessment of disease severity, and prediction of final outcome of these patients. The prospective study included 106 hospitalized patients with a clinical diagnosis of sepsis. Haematological parameters and CRP correlated with sepsis stage, and using ROC (Receiver operating characteristic) analysis were evaluated in the prediction of the final outcome of these patients. Among haematological parameters, patients with sepsis had a significantly higher proportion of total unsegmented neutrophil granulocytes, a lower percentage of lymphocytes, as well as a lower total platelet count ( $\mathrm{p}<0.05$ for all measurements). An excellent positive correlation was found between serum CRP concentration and disease stage $(\mathrm{r}=0.77)$. The best predictive value for the presence of sepsis was shown by CRP at the cutoff value of $165 \mathrm{mg} / \mathrm{L}$ (AUC 0.98), followed by the percentage of unsegmented neutrophil granulocytes for the cut-off value of 15.5 (AUC 0.67), and the percentage of lymphocytes less than 9.9 (AUC 0.66), platelets lower than $118 \times 10^{9} / \mathrm{L}$ (AUC 0.63). At the CRP cut-off value of $294.7 \mathrm{mg} / \mathrm{L}$ (AUC $0.84 ; 95 \%$ CI $0.74-0.93$ ), death could be predicted in $80.95 \%$ of patients with sepsis, with the sensitivity of $43.75 \%$ and the specificity of $89.71 \%$ ( $<<0.0001)$.
\end{abstract}

\footnotetext{
${ }^{1}$ Correspondence to:

Sehveta Mustafić , Department of Laboratory Diagnostics, University Clinical Centre Tuzla

E-mail: sehveta.mustafic@ukctuzla.ba
} 
By monitoring changes in haematological parameters and CRP concentration in combination with other clinical and laboratory indicators, disease severity and final outcome in patients with sepsis can be predicted.

Key words: CRP, lymphocytes, platelets, SIRS, sepsis,

\section{INTRODUCTION}

Sepsis is a life-threatening condition characterized by a systemic inflammatory response of the organism caused by a severe infection (Levy et al., 2003). It is associated with clinical and laboratory signs of disease such as fever, tachycardia, rapid breathing, changes in mental status, changes in the number of leukocytes, platelets, and many others. However, these parameters very often do not provide enough information for the differential diagnosis between non-bacterial and bacterial infections, infectious and non-infectious inflammatory conditions, as well as the severity of the disease itself. On the other hand, shortening the time required for diagnosis has been shown to be the most important component in reducing the risk of individual organ damage during sepsis and mortality associated with it (Jones et al., 2010). After a microbial infection or microbial intoxication, the immune system triggers a complex series of events that cause an immune response. The acute response of the organism in sepsis begins with the activation of the first line cells of defense of the immune system against pathogens-polymorphonuclear neutrophils, which make up 50-60\% of all circulating leukocytes. With any type of microbial trigger, the immune response is amplified over time and develops into an "excessive" immune response - systemic inflammatory response (SIRS), characteristic of sepsis, due to increased production of proinflammatory cytokines that include TNF (tumor necrosis factor), interleukin-1, interleukin-12, interferon gamma and interleukin-6. Total number of leukocytes is routinely recommended as the first screening marker for the presence of infection. Numerous studies have shown that increased number of leukocytes and absolute number of neutrophilic granulocytes, as well as a left shift are associated with sepsis (Wile et al., 2001). Also, numerous cytokines primarily TNF, IL-1 $\beta$ and IL- 6 mediate the initial response of the innate immune system to trauma or infection. TNF, IL-1 $\beta$ activate endothelial cells by attracting circulating polymorphonuclear leukocytesneutrophils (PMN) to the site of inflammation. They also penetrate the circulation causing fever and other systemic symptoms. IL-6 stimulates the liver to produce reactants of the acute phase of inflammation including CRP, and also leads to bone marrow stimulation and increased production of PMN. Depending on the degree of inflammation, this stimulation can cause PMN precursors to leave the bone marrow and enter the circulation even before they are fully mature. Increased release of total PMN, as well as an increase in the percentage of immature forms of PMN are one of the criteria for SIRS. As a consequence of the activation of circulating immune cells, the bone marrow in response to systemic infection releases immature granulocytes into the peripheral blood, which increases the ratio of immature/total granulocytes whose degree correlates well with the stage and severity of sepsis (Bender et al., 2008). 
Early diagnosis and adequate treatment of sepsis is a daily problem faced by doctors, especially in emergency rooms and intensive care units. Although there are numerous strategies for accessing and treating patients with sepsis, in order to improve survival rates, rapid and adequate diagnosis has proved to be the most important.

The aim of this study was to examine the importance of haematological parameters and CRP in the diagnosis of sepsis, assessment of disease severity and prediction of final outcome in these patients.

\section{MATERIAL AND METHODS PARTICIPANTS}

The prospective study included 106 patients hospitalized at the University Clinical Center Tuzla, with a clinical diagnosis of sepsis, with positive 2 or more criteria for SIRS. Based on the collected evidence of infection, $82(77.36 \%)$ patients were classified in the clinical group of patients with proven infection - patients with sepsis, while 24 (22.64\%) patients were a control group of patients without sepsis because no evidence of infection was found. Out of a total of 82 patients with sepsis, 52 of them $(63.41 \%)$ had a positive blood culture, and 30 (36.59\%) had a negative blood culture result. According to the ACCP / SCCM (American College of Chest Physicians / Society of Critical Care Medicine) (Anonymous, 1992) criteria for disease severity, $24(25.44 \%)$ patients had two or more positive signs of a systemic inflammatory response without clear evidence of infection and they were in the zero (0) stage of sepsis marked as SIRS. $43(45.58 \%)$ patients met the criteria for the first (I) stage of sepsis (SIRS + evidence of infection) marked as sepsis. In 31 (32.86\%) patients with SIRS and evidence of the presence of infection, organic damage was present and they were in the second (II) stage of sepsis-severe sepsis, while in 8 (8.06\%) patients, with sepsis and organic damage there was constant hypotension with perfusion disorder, and they were in a state of septic shock-stage III (III). Patients with proven malignancy, haematological patients on cytostatic therapy, as well as transplant patients were not included in the study.

\section{DESIGN AND PROCEDURES}

The clinical diagnosis of sepsis, according to ACCP / SCCM criteria (Anonymous, 1992) and clinical guidelines for sepsis (Dellinger et al., 2013), was made based on 2 or more positive indicators (signs) for SIRS. Patients diagnosed with suspected sepsis by the attending physician had undergone venepuncture for blood sample for blood culture directly on two different substrates for aerobic and anaerobic microorganisms and sent to the Department of Microbiology of the Polyclinic for Laboratory Diagnostics of the University Clinical Center Tuzla for analysis. At the same time, venous blood samples were taken from patients for haematological and biochemical tests and sent to the Department of Biochemistry of the Polyclinic for Laboratory Diagnostics of the University Clinical Center Tuzla. 


\section{MEASURES}

Haematological parameters: total leukocyte count, differential blood count and platelet count were determined on an haematological automatic counter Cell-Dyn Rubby (Abbot Diagnostic), after which microscopic specimens were made from the same blood sample, stained by the Gimza method and on which the proportion of non-segmented neutrophils and the percentages of all white blood cell parameters were determined microscopically under a magnification of 100. The CRP concentration was measured on a Dimension RxL (Siemens) biochemical analyzer. The obtained values were compared between patients with a clinical diagnosis of sepsis - SIRS and patients with a confirmed diagnosis of sepsis, as well as between patients with a positive blood culture and an isolated bacterial agent; and correlated with the stage (severity) of sepsis and evaluated in the prediction of the final outcome of these patients.

\section{STATISTICAL ANALYSIS}

Standard methods of descriptive statistics (range, median, arithmetic mean, standard deviation) were used in statistical data processing. The distribution of the variables was determined by the D'Agostino test, the Pearson omnibus test, and the Kolmogor-Smirnov normality test. Mean values are shown as mean \pm standard deviation in the case of a normal distribution, and in the case of a nonparametric distribution these values are shown as medians. Parametric and nonparametric tests (Student's t-test, Mann-Whitney test, Fisher's test and $\chi^{2}$ test with double and single orientation) were used to test the statistical significance of the difference between the samples; and the ANOVA test was used to calculate the relative difference in the variance distribution between the variables. Assessment of the diagnostic validity of the examined laboratory indicators in the diagnosis of sepsis in patients with the present systemic inflammatory response of the organism was made using the Receiver Operating Characteristic Curve (ROC curve) (Hanley and McNeil method). For the obtained ROC curves, the value of the area under curve (AUC) was calculated to determine the diagnostic accuracy of the examined clinical and laboratory indicators. The validity of the examined parameters is marked with the largest area under the curve, which implies the best sensitivity and specificity for a certain cut-off value. Univariate and multivariate logistic regression analysis was used to determine the predictive value of the examined variables for the presence or absence of sepsis, the presence / absence of positive blood culture, and mortality rate in these patients. The difference between the samples was considered significant when the result is $\mathrm{p}<0.05$. All data were analyzed using the statistical program GraphPad Prism, version 7 (San Diego, California, USA) and SPSS version 10 for Windows

\section{RESULTS}

Out of the total number of patients, 47 (49.82\%) were hospitalized at the Clinic for Resuscitation and Anesthesia, 30 (31.8\%) patients at the Clinic for Internal Medicine, 22 (23.32\%) patients were hospitalized at the Clinic for Infectious Diseases, while 7 (7.42\%) 
were placed in other hospital departments of the University Clinical Center Tuzla. The frequency of patients with sepsis was equally distributed between individual clinics ( $p>0.05$ ), and no significant difference was found in the positivity of blood culture in individual departments ( $>0.05)$. Both sexes were equally represented, 52 (49\%) male and $54(51 \%)$ female patients. Patients with sepsis were slightly older (57.84 \pm 1.65 years) compared to patients without sepsis $(49.54 \pm 4.2$ years $)(\mathrm{p}<0.05)$. Organic damage was present in $47.56 \%$ (39) versus $52.44 \%$ (43) of patients in whom no damage to any of the organ systems was found. Fatal outcome occurred in $15 \%$ of all patients with SIRS, in $19.51 \%$ of patients with sepsis, and in $28 \%$ of patients with positive blood culture. When it comes to the haematological parameters, patients with sepsis had a significantly higher proportion of total non-segmented neutrophils granulocytes, a lower percentage of lymphocytes, as well as a lower total platelet count ( $\mathrm{p}<0.05$ for all measurements) compared to patients without sepsis. Values of other monitored haematological parameters had an equal distribution between both groups ( $>0.05$ for all measurements). Lower total lymphocyte counts and lower platelet counts $(\mathrm{p}<0.05, \mathrm{p}<0.01$, respectively) were found in patients with positive blood culture compared to patients with negative blood culture. The values of other monitored haematological parameters had an equal distribution between patients with positive and negative blood culture ( $p>0.05$ for all measurements) (Table 1).

Table 1. Values of haematological parameters in sepsis

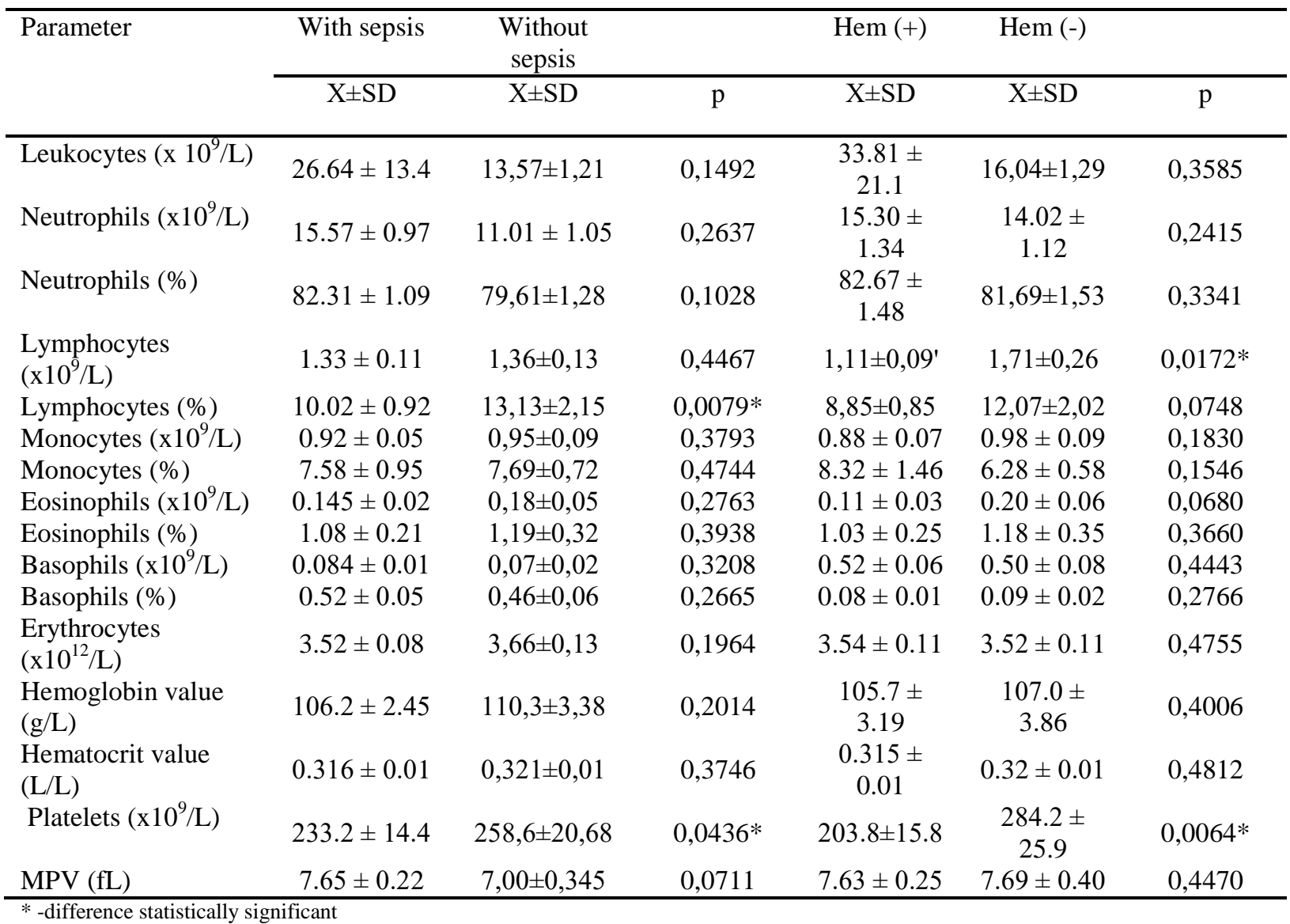

When it comes to the haematological parameters of white blood cell obtained by making a microscopic specimen and staining by the Gimza method, patients with sepsis had a 
significantly higher proportion of total non-segmented neutrophil granulocytes $22.13 \pm 1.51$ versus $16.75 \pm 2.96$ in patients without sepsis $(p<0.05)$, while other white blood cell parameters were equally distributed ( $\mathrm{p}>0.05$ for all measurements). Patients with sepsis also had a significantly higher serum CRP concentration of $240.65 \mathrm{mg} / \mathrm{L}$ (102.7-500.7), compared to patients without sepsis $104.55 \mathrm{mg} / \mathrm{L}(13.6-167.9)(\mathrm{p}<0.0001)$.

Also patients with sepsis and positive blood culture had a significantly higher concentration of CRP $269.2 \mathrm{mg} / \mathrm{L}$ (120.7-500.7) compared to patients with negative blood culture $213 \mathrm{mg} / \mathrm{L}$ (102.7-328.9) $(\mathrm{p}<0.0061)$. The concentration of CRP increased in each higher clinical stage compared to lower stages of the disease ( $p<0.001$ for all measurements), with the exception of the second and third stage, where there was no difference in the concentration of this parameter in these two stages $(\mathrm{p}>0.05)$ (Figure 1). An excellent positive correlation was found between serum CRP concentration and disease stage (Spearman correlation coefficient 0.77 ; $95 \%$ CI: 0.6725 to 0.8376 ; $\mathrm{p}<0.0001)$.

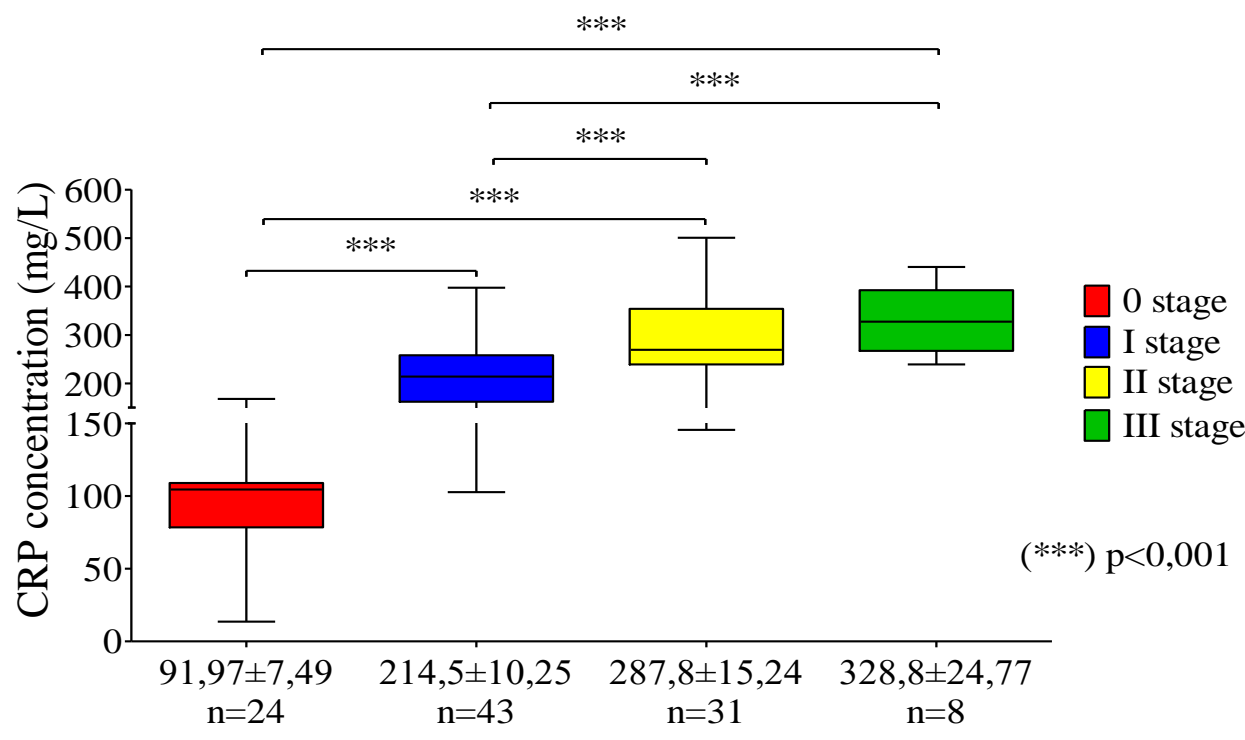

Figure 1. Values of CRP concentration in different stages of sepsis

Significantly higher CRP values were recorded in patients with a fatal outcome of 269.5 $\mathrm{mg} / \mathrm{L}$ (168-500.7) compared to patients without a fatal outcome of 164.95 (13.6-433) $(\mathrm{p}<0.0001)$. Using ROC analysis, the limit values of the predictive score of the presence of sepsis of those haematological parameters for which there was a difference between patients with present and absent sepsis (total unsegmented neutrophil granulocytes-microscopy, lymphocytes-differential blood count and platelets), as well as for CRP were determined. 
Table 2. Haematological parameters and c-reactive protein in prediction of sepsis

\begin{tabular}{lcccc}
\hline & \multicolumn{3}{c}{ Presence of sepsis } \\
\cline { 2 - 5 } & NNG*(\%) & $\begin{array}{c}\text { Lymphocytes } \\
\text { (DKS \%)** }\end{array}$ & $\begin{array}{c}\text { Platelets } \\
(\mathbf{x 1 0} / \mathbf{L})\end{array}$ & $\begin{array}{c}\text { CRP } \\
(\mathbf{m g} / \mathbf{L})\end{array}$ \\
\hline Cut-off value & 15.57 & 9.9 & 118.0 & 165.0 \\
area under the curve & $0.67 \pm 0.07$ & $0.66 \pm 0.06$ & $0.63 \pm 0.06$ & $0.98 \pm 0.01$ \\
p value & $<0.01$ & $<0.05$ & $<0.05$ & $<0.0001$ \\
95\% CI & 0.53 do 0.82 & 0.55 do 0.77 & 0.52 do 0.7 & 0.96 do 0.1 \\
Sensitivity & $58.33 \%$ & $60.0 \%$ & $24.39 \%$ & $84.15 \%$ \\
Specificity & $36.64 \%$ & $66.67 \%$ & $95.83 \%$ & $95.83 \%$ \\
PPV*** & $84.38 \%$ & $85.71 \%$ & $95.24 \%$ & $85.7 \%$ \\
NPV**** & $35.0 \%$ & $33.33 \%$ & $27.06 \%$ & $63.89 \%$ \\
Test accuracy & $65.38 \%$ & $61.54 \%$ & $40.57 \%$ & $86.79 \%$ \\
\hline$*$ NNG-unsegmented neutrophilic granulocytes-microscopy, $* *$ DKS-differential blood count, ***PPV- positive predictive value,
\end{tabular}

ROC analysis was also used to determine the limit values of the predictive score of the presence of positive blood culture of those hematological parameters, for which there was a difference between patients with positive and negative blood culture (lymphocytes-absolute number and platelets). A lymphocyte cut-off value (absolute number) below $0.81 \times 10^{9} / \mathrm{L}$ with the area under the AUC curve of 0.65 (95\% CI:0.5180 to $0.7741 ; \mathrm{p}<0.05)$ was obtained in patients with positive blood culture, with the sensitivity of $31.37 \%$ (95\% CI: $19.11 \%$ to $45.89 \%$ ) and the specificity of $79.31 \%$ (95\% CI:60.28\% to $92.01 \%$ ). The positive predictive value was $72.73 \%$ (95\% CI: $49.78 \%$ to $89.20 \%$ ), and the negative predictive value was $39.66 \%$ (95\% CI: $27.05 \%$ to $53.36 \%$ ). Using ROC analysis, a platelet limit value below 125.5 $\mathrm{x} 10^{9} / \mathrm{L}$ with an area under the AUC curve of 0.68 (95\% CI:0.5558 to $0.7948 ; \mathrm{p}<0.01$ ) was obtained in patients with a positive blood culture, with the sensitivity of $30.77 \%$ (95\% CI: $18.72 \%$ to $45.10 \%$ ) and the specificity of $90.0 \%$ (95\% CI:73.47\% to $97.89 \%$ ). The positive predictive value was $84.21 \%$ (95\% CI:60.40\% to $96.43 \%$ ), and the negative predictive value was $42.86 \%$ (95\% CI:30.46\% to $55.95 \%$ ). Using ROC analysis, the cut-off value of C-reactive protein above $239.7 \mathrm{mg} / \mathrm{L}$ with the area under the AUC curve of 0.76 (95\% CI:0.6519 to $0.8602 ; \mathrm{p}<0.0001$ ) was obtained in patients with positive blood culture. The sensitivity was $67.31 \%$ (95\% CI:52.90\% to $79.67 \%$ ) and the specificity was $76.67 \%$ (95\% CI:57.72\% to $90.07 \%$ ). The positive predictive value was $83.33 \%$ (95\% CI:68.63\% to 93.00), and the negative predictive value was $57.5 \%$ (95\% CI:40.89\% to 72.95). At the CRP cut-off of $294.7 \mathrm{mg} / \mathrm{L}$ (AUC $0.84 ; 95 \%$ CI 0.74 - 0.93), death could be predicted in $80.95 \%$ of patients with sepsis, with the sensitivity of $43.75 \%$ and the specificity of $89.71 \%$ (PPV 50.0\%; NPV 87.14\%) $(\mathrm{p}<0.0001)$.

\section{DISCUSSION}

Despite advances in diagnosis in better disease recognition, patients with sepsis are a very heterogeneous group of patients and the condition is very often difficult to recognize, especially in the early stages of the disease. The definition of sepsis, which includes SIRS+evidence or suspected infection, can be very broad and includes a large number of patients who do not have to develop sepsis. 
On the other hand, the transition from SIRS and sepsis to severe sepsis develops over time in different ways in different patients, so that even the development of severe sepsis may remain unrecognized until it is too late. Timely diagnosis of sepsis and early initiation of adequate therapy have been shown to lead to a better outcome in patients and a reduction in mortality caused by sepsis (Levy et al., 2010).

The results of our study, as in most cases of other authors, did not show a significant difference in the total number of leukocytes, however, patients with sepsis had a significantly higher proportion of total unsegmented neutrophil granulocytes compared to patients without sepsis $(\mathrm{p}<0.05)$, while this difference was not found in relation to a positive blood culture in patients with sepsis. In the study by Veeresh et al. (2012) no statistically significant difference was found in the total number of WBC and neutrophils between patients with sepsis with positive and negative blood cultures: WBC median $10.1 \times 10^{9} / \mathrm{L}(8.0-17.2)$ versus $11.06 \times 10^{9} / \mathrm{L}$ (6.75-13.28); neutrophil count $8.7 \times 10^{9} / \mathrm{L}$ (5.7-13.5) versus $8.51 \times 10^{9} / \mathrm{L}(4.55$ 10.85). Also, Longxiang et al. (2012) in the early diagnosis of sepsis versus SIRS found no difference in the total number of WBCs $\left(13.32 \times 10^{9} / \mathrm{L}\right.$ versus $\left.12.54 \times 10^{9} / \mathrm{L} ; \mathrm{p}=0.373\right)$, nor $\mathrm{did}$ Robriquet et al. (2013) between SIRS uninfected patients and patients with intrahospital proven infection WBC $12.68 \times 10^{9} / \mathrm{L}(8.8-13.5)$ versus $10.5 \times 10^{9} / \mathrm{L}(7.1-13.5)$ ( $\left.\mathrm{p}>0.05\right)$. In contrast, Huang et al. (2013) had a significant difference in PON values (neutrophil percentage) and did not find a significant difference in the total number of WBCs between patients with positive and negative blood cultures. PON to Hem (+) $0.86(0.80-0.917)$ vs. Hem (-) 0.82 (0.733-0.829); and WBC Hem (+) 9.87x 109/L (5.9-15.72) versus Hem (-) $9.71 \times 10^{9} / \mathrm{L}$ (6.27-14.07). Similar to our study, Yan et al (2013) found no significant difference in either total leukocyte count or percentage of immature neutrophils between patients with negative and positive blood cultures: WBC Hem $(+) 11.1 \pm 8.9 \times 10^{9} / \mathrm{L}$ vs. Hem () $10.6 \pm 7.9 \times 10^{9} / \mathrm{L}(\mathrm{p}=0.789)$; the percentage of granulocytes $84.6 \pm 28.2$ versus $85.1 \pm 19.3$ $(\mathrm{p}=0.792)$, and these parameters did not prove to be good predictors for a positive blood culture outcome: WBC AUC $0.42(\mathrm{p}=0.130)$ percentage of granulocytes AUC 0.508 $(\mathrm{p}=0.871)$. In our study, using ROC analysis, the limit value of total non-segmented neutrophilic granulocytes-microscopy above $15.57 \%$ (AUC 0.67 ) in the diagnosis of sepsis was obtained, with a test accuracy of $65.38 \%$, while they did not prove to be a significant predictor of positive blood culture findings. During bacterial infection, there is also a significant interaction between antigen presenting cells (ACPs) and lymphocytes, which are key effector cells in the body's acquired immune response. After a primary microbial infection, $\mathrm{T}$ cells do not have to receive an adequate stimulus from the ACP in terms of responding adequately to secondary infections (Murphey et al., 2004). The potential mechanism of loss of this $\mathrm{T}$ cell function during sepsis arises because the signal by the ACP is disrupted by inducing anergy, apoptosis, and a decrease in lymphocyte count. Although lymphocytopenia has been recognized as a marker of bacteremia, it has not been widely used as a marker for infection. The mechanism by which lymphocytopenia occurs in sepsis and septic shock involves the margination and redistribution of lymphocytes by the lymphatic system and is characterized by accelerated apoptosis. In patients with septic shock, lymphocyte apoptosis is greatly accelerated leading to constant lymphocytopenia associated with a poorer prognosis of the final outcome in these patients (Le Tulzo, 2002). 
We found a significantly lower percentage and absolute lymphocyte count in patients with sepsis compared to patients with noninfectious SIRS. With the lymphocyte proportion less than $9.9 \%$ in the differential blood count, the diagnosis of sepsis could be accurately predicted in $61.54 \%$ of SIRS cases (AUC 0.66), while a positive blood culture finding based on an absolute lymphocyte count of less than $0.81 \times 10^{9} / \mathrm{L}$ (AUC0.65) could be predicted in $72.73 \%$ of patients with sepsis. Initially, lymphocytopenia was described as a case report of toxic shock syndrome (Galus and Stern, 1998). Later, Zohorec (2001), in a prospective longitudinal study, demonstrated an association between disease severity and lymphocytopenia in oncology patients with severe sepsis and septic shock treated in the intensive care unit. Hawkins et al (2006) described the presence of T and B lymphocytopenia in Gram (+) and Gram (-) bacteremia, while Wyllie et al. (2004, 2005) confirmed the clinically useful application of lymphocytopenia in the prediction of bacteremia in two separate studies. By analyzing the hemogram, Juan et al (2013) proved a significant increase in the total number of leukocytes on account of neutrophils, with a statistically significant decrease in the number of lymphocytes and eosinophils in patients with sepsis. Cornelis et al. (2010) in their study compared the values of CRP, WBC, lymphocytes and neutrophil / lymphocyte ratios in patients with proven bacteremia. In their study, patients with a positive blood culture had lower lymphocyte values compared to patients with a negative blood culture $\left(0.8 \pm 0.5 \times 10^{9} / \mathrm{L}\right.$ versus $\left.1.2 \pm 0.7 \times 10^{9} / \mathrm{L} ; \mathrm{p}<0.0001\right)$; lymphocyte count also proved to be a good predictor of a positive blood culture outcome (AUC 0.73 ) for a cut-off value below $1.0 \times 10^{9} / \mathrm{L}$ with the sensitivity of $73.9 \%$ and the specificity of $57.6 \%$ (PPV $63.6 \%$, NPV $68.8 \%$ ). Significantly better predictive value for lymphocytes compared to total leukocyte count or neutrophil count, as it has been shown in our study results, was also found by Wyllie et al (2004) in the prediction of bacteremia. In a study by Liu et al. (2014), the number of lymphocytes in patients with sepsis correlated well with the severity of the disease, which makes them a good indicator for monitoring the condition of patients and the success of the applied therapy. In patients with non-infectious SIRS, sepsis and severe sepsis, they found a relative tendency to decrease the number, as well as the percentage of lymphocytes in the differential blood count: total lymphocyte count $1.53 \times 10^{9} / \mathrm{L}(0.89-1.88), 0.90 \times 10^{9} / \mathrm{L}(0.65-$ $1.42)$, and $0.80 \times 10^{9} / \mathrm{L}(0.50-1.12)$; percentage of lymphocytes $(0.225 \pm 0.122 ; 0.138 \pm 0.097$; and $0.106 \pm 0.070)$. Also, the number of lymphocytes, as in our study, was significantly lower in patients with a fatal outcome versus survivors: total lymphocyte count $0.90 \times 10^{9} / \mathrm{L}(0.50$ 1.29 ) versus $1.05 \times 10^{9} / \mathrm{L}(0.70-1.54)$; percentage of lymphocytes $0.123 \pm 0.098$ versus $0.143 \pm 0.097$.

Other white blood cells, with the help of various laboratory techniques and possibilities, can be used as special indicators of changes in the body during sepsis. Monocytes and macrophages, except in response to the innate immune system, are also involved in infection - induced anemia due to iron retention resulting in a sharp decrease in hemoglobin synthesis and hemoglobin content in reticulocytes, which may serve as an early indicator of monocyte macrophage activation (Franck et al., 2004). However, in our study, no significant difference was found in hemoglobin concentration and other red blood cell parameters between patients with sepsis and without sepsis, nor between patients with positive and negative blood cultures. 
This can be explained by the fact that patients were not previously specifically stratified and that the study included a large number of patients after surgical treatment, as well as a certain number of patients who receive dialysis, and in whom other possible causes of decreased hemoglobin were not excluded. Disseminated intravascular coagulation (DIC) is very common in patients with severe sepsis. Consumption of coagulation factors and platelets, together with inhibition of the fibrinolytic system, results in microvascular fibrin deposition. This compromises blood flow and participates in organ dysfunction characteristic of severe sepsis. Thrombocytopenia often occurs during severe sepsis and septic shock as a consequence of platelet consumption, splenic sequestration and microcirculation, peripheral destruction, and due to decreased production during hemophagocytosis (Thiolliere et al., 2013). The results of our study showed that patients with sepsis had a lower total platelet count compared to patients without sepsis as well as patients with a positive blood culture compared to patients with a negative blood culture. Decreased platelet counts can be used as an indicator of prediction of sepsis in patients with SIRS (AUC 0.63 \pm 0.06 ) and they can be used to predict a positive blood culture finding in patients with confirmed sepsis (AUC of $0.68 \pm 0.06)$.

Numerous experimental studies on animal models as well as human research have clearly shown that a sufficient number of platelets is an important determinant of the host immune response to infection. Platelets interact with bacteria through a large number of different molecular and cellular mechanisms. In the initial stage of uncomplicated sepsis, there is a decrease in platelet count as a result of increased concentrations of fibrinopeptide-A, aPAMP and quinocidin PF4 in the serum of these patients which then cause platelet degranulation (Michael, 2010). Recently, thrombocytopenia has been recognized as a significant, independent factor associated with infection and its mortality rate. Even in the absence of neutropenia, in elderly patients, thrombocytopenia was positively correlated with the frequency and severity of the disease in the case of bacterial pneumonia (Feldman et al., 1991). Similarly, Chang et al. (2000) demonstrated that thrombocytopenia is an independent predictor of infection severity and fatal outcome in liver transplant patients. Thrombocytopenia, in a study by Santoly et al. (2002), was shown to be an independent risk factor for bacterial infections with the sensitivity of $92 \%$ and the specificity of $76 \%$ (PPV $82 \%$, NPV 90\%) at the cut-off value $<50 \times 10^{9} / \mathrm{L}$, while Yoshida et al. (2005) demonstrated by multivariate analysis that decreased platelet count is an independent predictor of severe bacteremia. The better predictive value obtained in these studies compared to the results of our study is due to the use of a much lower cut-off value for platelets in these studies compared to ours (50 versus 118 ).

CRP is an acute phase protein member of the pentraxin protein family whose synthesis takes place in the liver induced by cytokines that are released during inflammation regardless of whether there is an infection or not. CRP plays an important role in the innate immune response to infection. It activates the complement system in the classical way through direct interaction with complement proteins. CRP can also activate phagocytic cells through interaction with the surface receptors of these cells. 
It is also involved in the removal of apoptotic cells from the body, the process of atherosclerosis, and it has been identified as a mediator of organic damage during myocardial infarction (Abij and Meinders, 2002). In our study, significant differences were found in CRP values in patients with sepsis compared to SIRS-positive patients but without confirmed sepsis as well as between patients with positive and negative blood culture $p<0.0001)$. CRP was also shown to be a significant predictor in the diagnosis of sepsis (AUC 0.98) at the cutoff value above $165.0 \mathrm{mg} / \mathrm{L}$, while a positive blood culture outcome could be predicted in $70.73 \%$ of sepsis patients (cut-off value 239.7 AUC 0.76). Robriquet et al. (2013) also found a statistically significant difference between SIRS of uninfected patients and patients with intrahospitally proven infection in CRP values of $173 \mathrm{mg} / \mathrm{L}$ (126-188) versus $57 \mathrm{mg} / \mathrm{L}$ (31105), and similar results were obtained by Longxiang et al. (2012) in a clinical study conducted to establish an early diagnosis of sepsis (CRP in sepsis $114 \mathrm{mg} / \mathrm{L}$ versus $83 \mathrm{mg} / \mathrm{L}$ in patients with SIRS, $\mathrm{p}<0.001$ ). A large number of studies, in addition to the statistically significant difference of this parameter in distinguishing SIRS from sepsis, also confirmed the significant predictive value of CRP in the early diagnosis of sepsis, as well as the prediction of a positive blood culture outcome. In the study by Jaimes et al. (2013), based on CRP values, SIRS $45.0 \mathrm{mg} / \mathrm{L}$ (10-99) could be distinguished from sepsis $131 \mathrm{mg} / \mathrm{L}$ (54-229), and at a slightly lower CRP cut-off value of $78 \mathrm{mg} / \mathrm{L}$ (AUC 0.71 ) they had the sensitivity of $66.6 \%$ and the specificity of $66.1 \%$. In a study by A-Jin Lia and Sang-Gyung Kim (2013) for the CRP cut-off value of $69.95 \mathrm{mg} / \mathrm{L}$, the sensitivity was $83.3 \%$ and the specificity was $52.5 \%$. The better predictive value of CRP obtained in our study can be explained by the fact of a higher cut-off value for CRP $(165.0 \mathrm{mg} / \mathrm{L})$ which resulted in higher sensitivity $(84.15 \%)$ and specificity $(95.83 \%)$ of this parameter in the diagnosis of sepsis. Slightly weaker, as in our study, but still a significant predictive value of CRP was found in the prediction of positive blood culture findings in studies by Veeresh et al. (2012) who compared CRP values between patients with sepsis with the positive blood culture of $212 \mathrm{mg} / \mathrm{L}(115-263)$ and the negative blood culture of $134 \mathrm{mg} / \mathrm{L}$ (68-238) where CRP at the cut-off value of $150 \mathrm{mg} / \mathrm{L}$ had the sensitivity of $69.6 \%$, and the specificity of $52.9 \%$ (AUC 0.64 ). Similar results were obtained by Huang et al. (2013); CRP in Hem (+) 110 mg/L (60.4-146.0) versus Hem (-) 74.0 $\mathrm{mg} / \mathrm{L}$ (34.0-120.0), where CRP at the same cut-off value of $150 \mathrm{mg} / \mathrm{L}$ had a sensitivity of $82 \%$, and a specificity of $35 \%$ (AUC 0.613 ) in the prediction of a positive blood culture finding. The concentration of CRP was increasing at each higher clinical stage compared to the lower stages of the disease, which allows assessing the severity of the disease based on this parameter, and distinguishing milder stages of sepsis from severe sepsis and septic shock associated with high mortality rates. Similar results were obtained by Canovic et al. (2006) for CRP values as the "gold standard" in the diagnosis of sepsis, for SIRS it was $119 \pm 89$ $\mathrm{mg} / \mathrm{L}$, for sepsis $159 \pm 51 \mathrm{mg} / \mathrm{L}$, for severe sepsis $254 \pm 181 \mathrm{mg} / \mathrm{L}$ and for septic shock $228 \pm 119$ $\mathrm{mg} / \mathrm{L}$. Significantly higher CRP values of $269.5 \mathrm{mg} / \mathrm{L}$ (168-500.7) were also recorded in patients with a fatal outcome compared to patients without a fatal outcome where CRP was $164.95 \mathrm{mg} / \mathrm{L}(13.6-433)(\mathrm{p}<0.0001)$, and cut-off positive correlation of the height of the serum concentration of CRP with the fatal outcome ( $r=0.46)$. In our study, at the obtained CRP cut-off value of $294.7 \mathrm{mg} / \mathrm{L}$ and AUC 0.84, a fatal outcome could have been predicted in $80.95 \%$ of patients with sepsis. 
Mortality in our study was $19.51 \%$ compared to mortality of SIRS-positive patients in ICU of $35.7 \%$ in the study by Rocker et al. (2004), mortality of 34,3\% found by Mhamed et al. (2004), and mortality of $22 \%$ in the study by Vincent et al. (1998). These differences in our results can be explained by the fact that our study included a number of patients who met the inclusion criteria for the study but who due to better general condition were not placed in ICU but in other hospital units, where mortality rate is generally lower.

\section{CONCLUSION}

Sepsis, severe sepsis, and septic shock are urgent medical conditions that require prompt treatment and patient care. This is a severe clinical condition very often accompanied by complications, in the form of failure of certain organs/organ systems, and often, especially severe sepsis and septic shock, result in death. During sepsis, a numerous of clinical and laboratory indicators change, which can be used for rapid identification of the disease and application of timely and adequate treatment, which proved to be decisive for the final outcome of these patients. By monitoring changes in hematological parameters and CRP concentration in combination with other clinical and laboratory indicators, disease severity and final outcome in patients with sepsis can be predicted.

\section{CONFIRMATION}

All authors confirm that there is no conflict of interest.

\section{REFERENCES}

1. Abij H and Meinders A (2002) C-reactive protein: history and revival. Eur J Int Med; 13:412-415.

2. A-Jin Lee and Sang-Gyung Kim (2013) Mean cell volumes of neutrophils and monocytes are promising markers of spsis in elderly patients. Blood Research; 48:193-197.

3. Anonymous (1992) American College of Chest Physicians/Society of Critical Care Medicine, Consensus Conference. Crit Care Med; 20(6):864-874.

4. Bender L, Thaarup J, Varming K, Krarup H, Ellermann-Eriksen S, Ebbesen F (2008) Early and late markers for the detection of early-onset neonatal sepsis. Dan. Med. Bull; 55(4):219-223.

5. Chang FY, Singh N, Gayowski T, Wagener MM, Mietzner SM, Stout JE, Marino IR (2000) Thrombocytopenia in liver transplant recipients: predictors, impact on fungal infections, and role of endogenous thrombopoietin. Transplantation; 69:70-75.

6. Cornelis PCJ, Paul TLW, Rejiv BM, Jacqueline JL, Tom P, Peter CW (2010) Lymphocytopenia and neutrophil-lymphocyte count ratio predict bacteremia better than conventional infection markers in an emergency care unit. Crit Care; 14(5): 192-196.

7. Čanović P, Nesić Lj, Tomović M, Čanović D (2006) Dijagnostički i prognostički značaj merenja prokalcitonina u toku septičkih stanja. Medicinski Časopis; 3:52-57. 
8. Dellinger RP, Mitchell ML, Andrew R, Annane D, Herwig G, Steven MO, et al. (2013) Surviving Sepsis Campaing: International Guidelines for Management of Severe Sepsis and Septic Shock:2012. Crit Care Med; 41(2):580-637.

9. Feldman C, Kallenbach JM, Levy H, Thorburn JR, Hurwitz MD, Koornhof HJ (1991) Comparison of bacteremic community-acquired lobar pneumonia due to Streptococcus pneumoniae and Klebsiella pneumoniae in an intensive care unit. Respiration; 58:265270.

10. Franck S, Linssen J, Messinger M, Thomas L (2004) Potential utility of Ret-Yin the diagnosis of iron restricted erythropoiesis. Clin. Chem.; 50(7):1240-1242.

11. Galus MA, Stern J (1998) Extreme lymphocytopenia associated with toxic shock syndrome. J Intern Med; 244:351-354.

12. Hawkins CA, Collignon P, Adams DN, Bowden FJ, Cook MC (2006) Profound lymphopenia and bacteremia. Intern Med $J ; 36: 385-388$.

13. Huang W, Fan Y, Ding-Xia S, You-Jiang Z, Yan-Ping L, Chao-jun L, at al. (2013) Predictive value of procalcitonin for excluding Bloodstream infection: results of a retrospective study and utility of a rapid, quantitative test for procalcitonin. Journal of International Medical Research; 41(5):167-168.

14. Jaimes AF, Rosa G, Valencia M, Arango C, Gomez CI, Garcia A, Ospina S, Osorno SC, Henao AI (2013) A latent class approach for sepsis diagnosis support use procalcitonin in the emergency room for diagnosis of severe sepsis. BMC Anesthesiology; 13(23):22472253.

15. Jones AE, Shapiro NI, Trzeciak S, et al. (2010) Emergency Medicine Shock Research Network (EMShockNet) Investigators: lactate clearance vs central venous oxygen saturation as goal of early sepsis therapy: A randomized clinical trial. JAMA; 303:739746.

16. Juan CAB, Constantino TC, Carlos GB, Pérez-Griera J, Victoria A, Amadeo AQ, Juan RC, Carlos PO, Ferran B (2013) Association of $\gamma \delta$ T Cells with Disease Severity and Mortality in Septic Patients. Clin Vaccine Immunol; 20(5):738-746.

17. Le Tulzo Y, Pangault C, Gacouin A, Guilloux V, Tribut O, Amiot L, Tattevin P, Thomas R, Fauchet R, Drenou B (2002) Early circulating lymphocyte apoptosis in human septic shock is associated with poor outcome. Shock; 18:487-494.

18. Levy MM, Fink MP, Marshall JC, et al. (2003) "2001 SCCM/ESICM/ACCP/ATS/SIS International Sepsis Definitions Conference“. Crit. Care Med; 31(4):1250-1256.

19. Levy MM, Dellinger RP, Townsend SR, et al. (2010) Surviving Sepsis Campaing: The Surviving Sepsis Campaing: Results of an international guideline-based performance improvement program targeting severe sepsis. Crit Care Med; 38:367-374.

20. Liu H, Liu G, Tian Z (2014) Changes in blood lymphocytes in sepsis patients. Zhonghua Wei Zhong Bing Ji Jiu Xue; 26(3):148-152.

21. LonxiangS, Bingchao H, Changting L, liling L, Zhaoxu J, Jie D, Peng Y, Yhanhong j, Dan F, Lixin X (2012) Value of soluble TREM-1, procalcitonin, and C-rective protein serum levels as biomarkers for detecting bacteremia among sepsis patients with new fever in intensive care units: a prospective cohort study. BMC Infectious Diseases; 12:157-159. 
22. Mhamed SM, Nizar A, Nabil F, Naceur SS, Mohamed SA (2004) Individual Organ SOFA Score and Prediction of Mortality in ICU. Anesthesiology; 101:A408.

23. Michael RY (2010) Platelets in defense against bacterial pathogens. Cell Mol Life Sci; 67(4): 525-544.

24. Murphey ED, Lin CY, McGuire RW, Toliver-Kinsky T, Herndon DN, Sherwood ER (2004) Diminished bacterial clearance is associated with decreased IL-12 and interferon$\gamma$ production but a sustained pro-inflammatory response in a murine model of post-septic immunosuppression. Shock; 21:415-425

25. Robriquet L, Sejourne C, Kipnis E, Dherbomez M, Fourrier F (2013) A composite score combining procalcitonin, $\mathrm{C}$ reactive protein and temperature has a high positive predictive value for the diagnosis of intensive care-acquired infections. BMC Infectious Diseases; 13:159.

26. Rocker G, Cook D, Sjokvist P, Weaver B, Finfer S, McDonald E, et al. (2004) Clinician predictions of intensive care unit mortality. Crit Care Med; 32(5):1149-1154.

27. Santolaya ME, Alvarez AM, Aviles CL, Becker A, Cofre J, Enriquez N, O'Ryan M, Paya E, Salgado C, Silva P, Tordecilla J, Varas M, Villarroel M, Viviani T, Zubieta M (2002) Prospective evaluation of a model of prediction of invasive bacterial infection risk among children with cancer, fever, and neutropenia. Clin Infect Dis; 35:678-683.

28. Thiolliere F, Serre-Sapin AF, Reignier J, Benedit M, Constantin JM et al. (2013) Epidemiology and outcome of thrombocytopenic patients in the intensive care unit: results of a prospective multicenter study. Intensive Care Med; 39:1460-1468.

29. Veeresh KP, Jaymin BM, Francois DV, Suresh KB (2012) Associations Between Procalcitonin and Markers of Bacterial Sepsis. Medicine(Kaunas); 48(8):383-387.

30. Vincent, Jean-Luis, de Mendonca, Arnaldo, Cantraine F, Moreno R, Takala J, et al. (1998) Use of the SOFA score to assess the incidence of organ dysfunction/failure in intensive care units: Results of multicenter, prospective study. Crit care Med; 26(11):1793-1800.

31. Yan S, Bin D, Ying-Chun X, Xi R, Wei D, Yao W (2013) Early changes of procalcitonin predict bacteremia in patients With intensive care unit-acquired new fever. Chin Med J; 126(10):1832-1837.

32. Yoshida T, Tsushima K, Tsuchiya A, Nishikawa N, Shirahata K, Kaneko K, Ito K, Kawakami H, Nakagawa S, Suzuki T, Kubo K, Ikeda S (2005) Risk factors for hospitalacquired bacteremia. Intern Med; 44:1157-1162.

33. Wile MJ, Homer LD, Gaehler S, Philips S, Millan J (2001) Manual differential cell count help predict bacterial infection. A multivariate analysis. Am J Clin Pathol; 115:644-649.

34. Wyllie DH, Bowler IC, Peto TE (2004) Relation between lymphopenia and bacteremia in UK adults with medical emergencies. J Clin Pathol; 57:950-955.

35. Wyllie DH, Bowler IC, Peto TE (2005) Bacteremia prediction in emergency medical admissions: role of C reactive protein. J Clin Pathol; 58:352-356.

36. Zahorec R (2001) Ratio of neutrophil to lymphocyte counts - rapid and simple parameter of systemic inflammation and stress in critically ill. Bratisl Lek Listy; 102:5-14. 